Geringere Mortalität

\section{Ist die Schlafapnoe für Ältere sogar vorteilhaft?}

Lavie P et al. Unexpected survival advantage in elderly people with moderate sleep apnea. J Sleep Res. 2009;18:397-403

\section{Hintergrund und Fragestellung}

Die obstruktive Schlafapnoe (OSA) ist mittlerweile ein anerkannter kardiovaskulärer Risikofaktor [1]. Besonders häufig ist die Schlafapnoe jenseits des 65. Lebensjahrs [2, 3].

Die klinische Bedeutung der Schlafapnoe bei älteren Patienten wird aber unterschiedlich bewertet. Daher wurde die Mortalität von älteren Patienten mit und ohne Schlafapnoe untersucht.

\section{Methodik}

In die retrospektive Studie waren 601 Menschen in einem Alter von mindestens 65 Jahren eingeschlossen, bei denen mittels Schlaflaboruntersuchung die Diagnose einer Schlafapnoe gestellt worden war. Die Mortalitätsraten wurden einem Kontrollkollektiv (Zufallsstichprobe aus der Allgemeinbevölkerung) gegenübergestellt, das bezüglich Alter, Geschlecht und ethnischer Zugehörigkeit abgeglichen war.

Die Patienten wurden im Mittel über 5,17 Jahre nachverfolgt. Das mittlere Al-

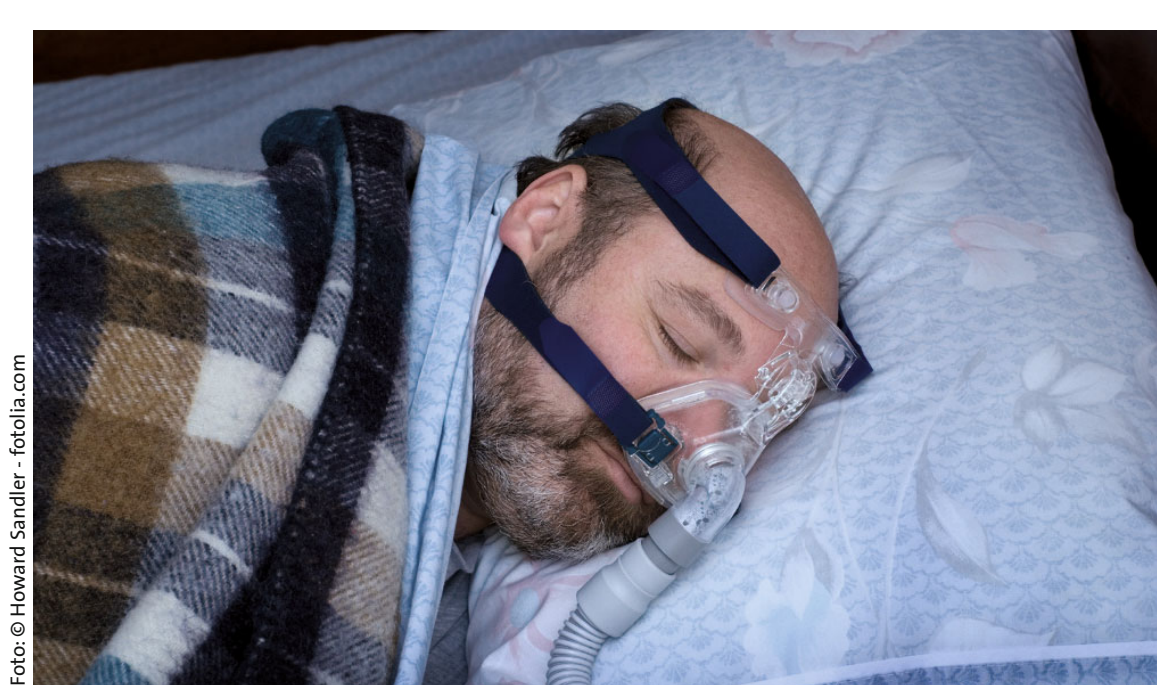

Eigentlich hat die Schlafapnoe einen negativen Einfluss auf die Lebenserwartung. Bei

älteren Patienten scheint dies aber anders zu sein. mittlere Body-Mass-Index (BMI) 30,4 $\mathrm{kg} / \mathrm{m}^{2}$ und der durchschnittliche Respiratory Disturbance Index (RDI) 28,9/h.

\section{Ergebnisse}

Die Gesamtsterblichkeit im Beobachtungszeitraum lag bei $12,3 \%$. Die standardisierte Mortalitätsrate (SMR) im Schlaflaborkollektiv betrug insgesamt 0,67. Differenziert nach RDI-Klassen ergab sich folgendes Bild:

- Die SMR war in der Gruppe mit einem RDI > 40/h am größten $(0,97)$, aber sie war nur in etwa so hoch wie in der Kontrollgruppe.

- Die zweithöchste SMR $(0,71)$ hatte die Gruppe mit einem RDI $<20 /$ h. Sie war bereits niedriger als in der Kontrollgruppe.

- Die niedrigste SMR $(0,42)$ wurde in der Gruppe mit einem RDI von 20$40 / \mathrm{h}$ gefunden. Sie war viel niedriger als in der Kontrollgruppe.

Die Regressionsanalyse belegte darüber hinaus, dass nicht die Schwere der
Schlafapnoe, sondern das Ausmaß der Komorbiditäten sowie eine verlängerte Schlaflatenz die Mortalitätsrate erhöhte.

\section{Schlussfolgerung}

Der nachgewiesene Überlebensvorteil von älteren Menschen mit mittelgradig ausgeprägter Schlafapnoe könnte auf kardioprotektive Effekte durch eine chronische intermittierende Hypoxie zurückgeführt werden.

\section{Kommentar}

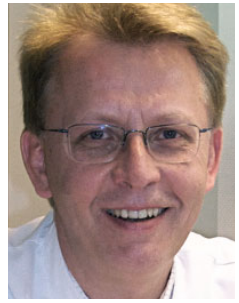

Prof. Dr. med.

Kurt Rasche,

Wuppertal

Diese Ergebnisse sind ausgesprochen interessant. Schon das Ergebnis, dass ältere Menschen mit schwergradiger Schlafapnoe keine Sterblichkeitssteigerung gegenüber einem Kontrollkollektiv zeigen, ist beachtenswert, umso mehr der Überlebensvorteil bei mittelgradig ausgeprägter Schlafapnoe.

Die Autoren präferieren als Erklärung die „verzögerte ischämische Präkonditionierung“, sozusagen eine Art „Hyposensibilisierung", um diesen Begriff aus der Allergologie zu entleihen, gegenüber der Hypoxämie, die dann im höheren Lebensalter zu einem Überlebensvorteil führt [4]. Diese Hypothese ist mit Sicherheit noch nicht genügend wissenschaftlich belegt, aber höchst interessant.

\section{Fazit}

Für die tägliche schlafmedizinische Praxis bedeutet dies, dass wir die Therapieindikation bei älteren Schlafapnoepatienten, die vielleicht sogar noch asymptomatisch sind, eventuell noch kritischer stellen müssen.

\section{Literatur}

1. Malhotra A et al. Lancet $2002 ; 360: 237-245$

2. Mehra et al. J Am Geriatr Soc. 2007;55:13561364

3. Young T. et al. Arch Intern Med. 2002;162:863900

4. Lavie L et al. Med Hypotheses 2006;66:10691073 